\title{
Thyrotoxic periodic paralysis as the first manifestation of a thyrotropin-secreting pituitary adenoma
}

\author{
Theodora Pappa ${ }^{1}$, Labrini Papanastasiou ${ }^{1}$, Athina Markou ${ }^{1}$, Ioannis Androulakis ${ }^{1}$, \\ George Kontogeorgos ${ }^{2}$, Andreas Seretis ${ }^{3}$, George Piaditis ${ }^{1}$ \\ ${ }^{1}$ Department of Endocrinology and Diabetes Center, ${ }^{2}$ Department of Pathology, ${ }^{3}$ Department of Neurosurgery, Athens \\ General Hospital “G. Gennimatas”, Athens, Greece
}

\begin{abstract}
Thyrotoxic Periodic Paralysis (TPP) is a rare manifestation of hyperthyroidism characterized by muscle weakness and hypokalemia. Thyroid-Stimulating Hormone (TSH)-secreting pituitary adenoma is a rare cause of hyperthyroidism. Even more rare is the occurrence of TPP as the first manifestation of a TSH-secreting pituitary adenoma. We report a 31-year-old Asian male patient suffering from TPP caused by a TSH-secreting adenoma, who was evaluated for persistent episodes of muscle paralysis. Laboratory investigation revealed hypokalemia as well as elevated levels of both thyroid hormones and TSH. The Magnetic Resonance Imaging (MRI) of the pituitary gland revealed a microadenoma, thus suggesting the presence of a TSH-secreting adenoma. The patient underwent transphenoidal resection and the pathological investigation confirmed the diagnosis of TSH-secreting pituitary adenoma. After the adenomectomy and the restoration of euthyroidism, the patient did not experience any episode of hypokalemic paralysis or weakness. Despite its rarity, TSH-secreting pituitary adenoma should be included in the differential diagnosis of TPP.
\end{abstract}

Key words: Hypokalemia, Pituitary adenoma, Thyrotoxic periodic paralysis, Thyrotropin-secreting pituitary adenoma.

\section{INTRODUCTION}

Thyrotoxic Periodic Paralysis (TPP) is a rare syndrome characterized by episodes of muscle paralysis associated with hypokalemia and hyperthyroidism. It is considered a sporadic disease affecting most frequently

\footnotetext{
Address for correspondence:

Papanastasiou Labrini, 7 Mantzarou Str., 15452 P. Psychiko, Athens, Greece, Tel: +306938601745, Fax: +302106990702, +302107779146, e-mail: linapapan@yahoo.gr

Received 19-09-09, Revised 10-11-09, Accepted 01-12-09
}

Asian males suffering from hyperthyroidism in the second and third decade of their life. ${ }^{1}$ The incidence of TPP in Chinese and Japanese thyrotoxic patients has been reported at $1.8 \%$ and $1.9 \%$, respectively, ${ }^{2,3}$ whereas in North Americans at 0.1-0.2\%. ${ }^{4}$ A male predominance has been widely described, with an overall male to female ratio ranging from $17: 1$ to $70: 1 .^{2,3,5}$ The severity of the episodes ranges from mild

Funding: This research did not receive any specific grant from any funding agency in the public, commercial or not-for-profit sector. 
weakness to complete flaccid paralysis. Cardiac arrythmias due to hypokalemia and respiratory failure are the two potentially life-threatening complications of this condition. The differential diagnosis includes Familial Hypokalemic Periodic Paralysis (FHPP), an autosomal dominant condition that affects mainly Caucasians in the absence of hyperthyroidism. ${ }^{6}$

On the other hand, the Thyroid Stimulating Hormone (TSH)-secreting pituitary adenoma as a cause of hyperthyroidism is a rare clinical entity. ${ }^{7}$ It accounts for less than $1 \%$ of all functioning pituitary adenomas $^{8}$ and even less than $1 \%$ of all cases of hyperthyroidism. The majority of them are macroadenomas usually manifesting with symptoms of tumor expansion. ${ }^{9}$ Inappropriately normal or high serum TSH concentrations and high serum thyroxine $\left(\mathrm{T}_{4}\right)$ and triiodothyronine $\left(\mathrm{T}_{3}\right)$ concentrations are the characteristic biochemical abnormalities observed in patients with hyperthyroidism caused by a TSHsecreting adenoma.

TPP is usually associated with Graves' disease. ${ }^{10}$ It is less commonly associated with thyroiditis, ${ }^{11}$ toxic adenoma, ${ }^{12} \mathrm{~T}_{4}$ ingestion and iodine excess. ${ }^{13,14}$ To date, only three cases of TSH-secreting adenoma presenting with TPP have been reported. ${ }^{15-17}$

In this article we describe a patient suffering from TPP due to a TSH-secreting adenoma. Our case is the first ever described in which a TSH-secreting microadenoma was discovered during investigations for the etiology of TPP.

\section{PATIENT'S DESCRIPTION AND METHODOLOGY}

A 31-year-old Syrian male presented to our outpatient department with persistent hypokalemia associated with repeated episodes of severe muscle weakness. Specifically, the patient reported several episodes of flaccid paralysis of the lower limbs over the past two years precipitated by ingestion of carbohydrate-rich meals. For this reason, he had repeatedly sought medical help, whereupon persisting hypokalemia was diagnosed. Serum potassium levels $\left(\mathrm{K}^{+}\right)$measured during episodes ranged from 2.5 to $2.8 \mathrm{mmol} / \mathrm{L}$, whereas urinary potassium excretion was low. The patient received potassium supplementation with subsidence of his symptoms and was instructed to take potassium tablets on offset of attacks of muscle weakness and lower limb paralysis. He had no significant medical history and had received no medication; his family history was negative for FHPP. No heat intolerance, weight loss, change in bowel habits, other symptoms of hyperthyroidism or headaches were reported.

On admission, the patient was in a good nutritional state (BMI: 25.4) with normal body temperature $\left(36.7^{\circ} \mathrm{C}\right)$ and blood pressure $(125 / 70 \mathrm{mmHg})$. There was no exophthalmos or lid lag, no moist skin or tremor or other signs of thyrotoxicosis, except for an elevated heart rate (102 bpm) and slightly increased tendon reflexes. Visual acuity and visual field examination were normal. The thyroid gland appeared diffusely enlarged, soft and tender without palpable nodules. Chest X-ray was normal and electrocardiogram showed mild sinus tachycardia. Serum $\mathrm{K}^{+}$levels were slightly decreased (3.3, Normal Range NR: 3.5-5 mmol/L), whereas the rest of his biochemistry and hematology was within normal range. Thyroid function tests revealed elevated levels of serum total $\mathrm{T}_{3}$ and free thyroxine $\mathrm{fT}_{4}(2.93$, NR: $0.89-244 \mathrm{nmol} / \mathrm{L}$ and 27.4 , NR: $9.0-21.0 \mathrm{pmol} / \mathrm{L}$, respectively) with inappropriately elevated TSH levels (5.55, NR 0.35-4.94 mU/L).

Because of suspicion of a TSH-secreting adenoma the patient was further evaluated. The rest of the anterior pituitary hormonal function was unremarkable and thyroid antibodies were negative. Serum $\alpha$-subunit levels were within normal limits $(0.62$, NR $<0.8 \mu \mathrm{g} / \mathrm{L})$. Thyroid ultrasound revealed diffuse enlargement of the thyroid gland and a Tc-99m scan showed increased uptake. Pituitary magnetic resonance imaging (MRI) showed a $3 \mathrm{~mm}$ lesion on the right side of the gland with signs of previous hemorrhage within it (Figure 1).

It should be mentioned that an electromyogram to evaluate muscle-fiber conduction velocity was not performed, since potassium supplementation rapidly restored potassium levels and resolved episodes of muscle paralysis.

The clinical picture of hypokalemic periodic paralysis, the biochemical findings of central hyperthyroidism and the positive pituitary imaging led to the diagnosis of a TSH-secreting pituitary microadenoma presenting with thyrotoxic periodic paralysis.

Treatment with antithyroid drugs (methimazole 


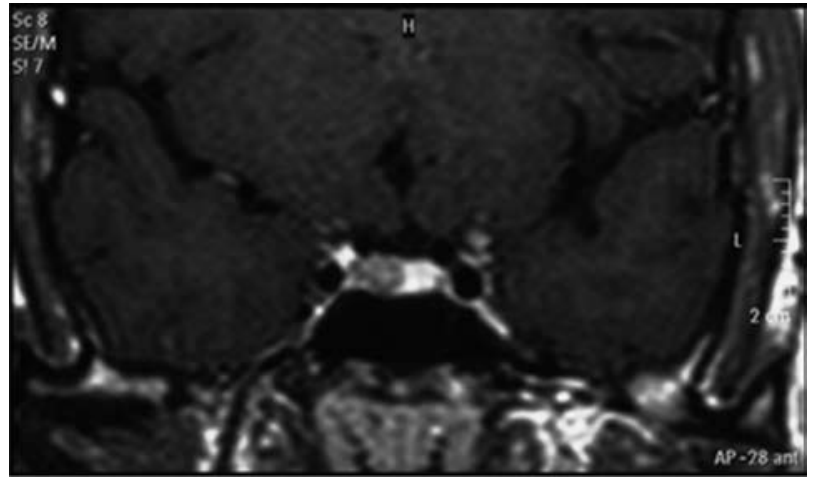

Figure 1. Pituitary MRI (coronal plane) revealing a $3 \mathrm{~mm}$ lesion in the right side of the pituitary.

20mg 2 times/day) and $\beta$-blockade (propranolol 20mg 2 times/day) was instituted to render the patient euthyroid pre-operatively, as well as potassium supplementation to maintain normal potassium levels. One month later the patient was clinically and biochemically euthyroid. Medical treatment was discontinued and successful transphenoidal adenomectomy was performed. Two weeks after surgery the TSH level was $1.25 \mathrm{mU} / \mathrm{L}$ and fT4 $14.03 \mathrm{pmol} / \mathrm{L}$.

Histology revealed a chromophobe adenoma, and immunohistochemistry was positive for $\beta$-TSH subunit, $\beta$-LH subunit and $\alpha$-SU and negative for GH, PRL, ACTH and $\beta$-FSH subunit, whereas Ki-67 was $3 \%$ and immunostaining for $\mathrm{p}-53$ was positive.

Two months later the patient underwent the first postoperative evaluation. He was in excellent clinical condition reporting no recurrence of episodes of paralysis or muscle weakness, with TSH level of $1.38 \mathrm{mU} / \mathrm{L}$ and $\mathrm{fT} 4$ level of $13.1 \mathrm{pmol} / \mathrm{L}$. The pituitary MRI scan did not show residual disease and anterior pituitary function profile was normal. In order to confirm and establish a total cure, a T3 suppression test was performed, i.e. T3 $25 \mathrm{mg} 3$ times/day was administered orally for seven days leading to suppression of TSH to $<0.01 \mathrm{mU} / \mathrm{L}$.

\section{DISCUSSION}

The TSH-secreting adenoma is a very rare cause of hyperthyroidism and TPP an uncommon first manifestation of hyperthyroidism. The history, physical examination and laboratory data of our case were consistent with TPP due to a TSH-secreting adenoma.
It is the fourth case in the literature of a TSH-secreting adenoma presenting with TPP and the first one associated with microadenoma.

Consistent with the diagnosis of TPP are the patient's demographic data (an oriental male in his 30s), the absence of a family history of periodic paralysis and the resolution of the attacks of periodic paralysis after restoration of potassium levels and euthyroidism. The subtle clinical features of hyperthyroidism, the diffusely enlarged thyroid gland, the increased uptake in the Tc-scan and the lack of ophthalmopathy observed in our patient are frequently reported in patients with TSH-secreting pituitary tumors. ${ }^{718}$ The normal alpha-subunit levels is possibly explained by the small size and the good differentiation of the adenoma. Because of the small size of the adenoma, the patient had no visual defects, no headaches and no hypopituitarism, all signs of pituitary tumor expansion commonly seen in patients with TSH-secreting pituitary tumors, as most of them are macroadenomas at the time of diagnosis. ${ }^{9}$ The patient's predisposition to TPP -because of his Asian origin- has contributed to the early diagnosis of thyrotoxicosis; consequently, the recognition of the TSH-secreting adenoma at an early stage possibly explains the small size of the adenoma in this patient.

The treatment of choice for TSH-secreting adenomas is transphenoidal adenomectomy. ${ }^{19}$ In certain cases, administration of somatostatin analogs (octreotide) and/or dopamine agonists (bromocryptine, cabergoline) have been successfully used to reduce TSH secretion and tumor size preoperatively and thus improve surgical outcome. ${ }^{8,20,21}$ Treatment with thionamides to prevent thyroid hormones synthesis should be used with caution, as it may further increase TSH secretion and accelerate tumor growth. Our patient was treated with antithyroid drugs and $\beta$-blockers to restore euthyroidism preoperatively without the need for additional therapy (octreotide or bromocryptine). Thereafter, a transphenoidal selective adenomectomy was successfully performed as documented by the normal pituitary function and MRI scan post-operatively, the absence of episodes of TPP and the complete suppression of TSH following the T3 suppression test.

The pathogenesis of TPP remains obscure; its main 
feature is hypokalemia induced by a massive shift of potassium from the extracellular into the intracellular compartment, mostly into the muscles, and it is attributed to an increase in the number and activity of the Na-K-ATPase pump. ${ }^{22,23}$ It has been demonstrated that adrenergic stimulation increases intracellular cAMP levels thus stimulating the Na-K-ATPase and promoting cellular potassium uptake. Elevated thyroid hormones increase the activity of Na-K-ATPase either directly or by increasing the number and sensitivity of $\beta$-receptors, inducing a hyperadrenergic state and consequently further potassium uptake. ${ }^{24}$ This suggests that thyrotoxicosis per se is implicated in the pathogenesis of TPP regardless of its etiology and explains the effect of treatment with $\beta$-blockers in blunting and preventing episodes of periodic paralysis. Insulin has also been shown to increase Na-K-ATPase activity, this explaining the offset of TPP attacks after carbohydrate-rich meals. ${ }^{25}$

The role of genetics in identifying patients susceptible to TPP has especially been studied among Asian populations. Specifically, in patients with TPP a higher incidence of HLA B46, DR9 and DQB1*0303 has been documented in Hong Kong Chinese patients and HLA A2, BW22, AW19, B17 and DRW8 in Singapore Chinese and Japanese patients. ${ }^{26-28}$ Moreover, an association between certain single-nucleotide polymorphisms (SNPs) of the L-type calcium channels a1-subunit Cav1.1 has been reported in Southern Chinese TPP patients. ${ }^{29}$ However, no specific association of HLA haplotypes or SNPs polymorphisms and predisposition to TPP has been documented.

In this report, we describe the fourth case of TPP as the first manifestation of a TSH-secreting adenoma. In addition, this is the first TSH microadenoma presenting with TPP. In clinical practice, thyrotropin-secreting pituitary adenoma should be included in the differential diagnosis of hyperthyroidism, especially when unsuppressible TSH levels are detected. The prompt diagnosis and treatment of the cause of hyperthyroidism in such cases leads to complete resolution of TPP thus preventing its potentially lethal cardiopulmonary complications.

\section{REFERENCES}

1. Kung AW, 2006 Clinical review: Thyrotoxic periodic paralysis: a diagnostic challenge. J Clin Endocrinol Metab 91: 2490-2495.

2. McFadzean AJS, Yeung R, 1967 Periodic paralysis complicating thyrotoxicosis in Chinese. Br Med J 1: 451-455.

3. Okinaka S, Shizume K, Lino S, et al, 1957 The association of periodic paralysis and hyperthyroidism in Japan. J Clin Endocrinol Metab 17: 1454-1459.

4. Kelley DE, Gharib H, Kennedy FP, et al, 1989 Thyrotoxic periodic paralysis. Report of 10 cases and review of electromyographic findings. Arch Intern Med 149: 2597-2600.

5. Ko GT, Chow CC, Yeung VT, et al, 1996 Thyrotoxic periodic paralysis in a Chinese population. QJM 89: 463-468.

6. Links TP, Smit AJ, Molenaar WM, et al, 1994 Familial hypokalemic periodic paralysis. Clinical, diagnostic and therapeutic aspects. J Neurol Sci 122: 33-43.

7. Beck-Peccoz P, Brucker-Davis F, Persani L, et al, 1996 Thyrotropin-secreting pituitary tumors. Endocr Rev 17: 610-638.

8. Socin HV, Chanson P, Delemer B, et al, 2003 The changing spectrum of TSH-secreting pituitary adenomas: diagnosis and management in 43 patients. Eur J Endocrinol 148: 433-442.

9. Beck-Peccoz P, Persani L, 2008 Thyrotropin-secreting pituitary adenomas. In: Thyroid disease manager. Available at: http://www.thyroidmanager.org.

10. Ober KP, 1992 Thyrotoxic periodic paralysis in the United States. Report of 7 cases and review of the literature. Medicine (Baltimore) 71:109-120.

11. Gluck FB, Nusynowitz ML, Plymate S, 1975 Chronic lymphocytic thyroiditis, thyrotoxicosis, and low radioactive iodine uptake. Report of four cases. N Engl J Med 293: 624-628.

12. Tagami T, Usui T, Shimatsu A, Naruse M, 2007 Toxic thyroid adenoma presenting as hypokalemic periodic paralysis. Endocr J 54: 797-803.

13. Layzer RB, Goldfield E, 1974 Periodic paralysis caused by abuse of thyroid hormone. Neurology 24: 949-952.

14. Tran HA, 2005 Inadvertent iodine excess causing thyrotoxic hypokalemic periodic paralysis. Arch Intern Med 165: 831-834.

15. Kiso Y, Yoshida K, Kaise K, et al, 1990 A case of thyrotropin-secreting tumor complicated by periodic paralysis. Jpn J Med 29: 399-404.

16. Alings AM, Fliers E, de Herder WW, et al, 1998 A thyrotropin-secreting pituitary adenoma as a cause of thyrotoxic periodic paralysis. J Endocrinol Invest 21: 703-706.

17. Hsu FS, Tsai WS, Chau T, et al, 2003 Thyrotropin-secreting pituitary adenoma presenting as hypokalemic periodic paralysis. Am J Med Sci 325: 48-50.

18. Brucker-Davis F, Oldfield EH, Skarulis MC, et al, 1999 Thyrotropin-secreting pituitary tumors: diagnostic criteria, thyroid hormone sensitivity, and treatment outcome in 
25 patients followed at the National Institutes of Health. J Clin Endocrinol Metab 84: 476-486.

19. Beck-Peccoz P, Persani L, 2008 Thyrotropinomas. Endocrinol Metab Clin North Am 37: 123-34.

20. Kienitz T, Quinkler M, Strasburger CJ, Ventz M, 2007 Long-term management in five cases of TSH-secreting pituitary adenomas: a single center study and review of the literature. Eur J Endocrinol 157: 39-46.

21. Colao A, Filippella M, Pivonello R, et al, 2007 Combined therapy of somatostatin analogues and dopamine agonists in the treatment of pituitary tumours. Eur J Endocrinol 156: Suppl 1: S57-63.

22. Chan A, Shinde R, Chow CC, et al, 1991 In vivo and in vitro sodium pump activity in subjects with thyrotoxic periodic paralysis. Br Med J 303: 1096-1099.

23. Lo CS, Edelman IS, 1976 Effect of triiodothyronine on the synthesis and degradation of renal cortical $\mathrm{Na}+$ $\mathrm{K}+$ adenosine triphosphatase. J Biol Chem 251: 78347840 .

24. Layzer RB, 1982 Periodic paralysis and the sodium- potassium pump. Ann Neurol 11: 547-552.

25. Hundal HS, Marette A, Mitsumoto Y, et al, 1992 Insulin induces translocation of the $\alpha 2$ and $\beta 1$ subunits of the $\mathrm{Na}+/ \mathrm{K}(+)$-ATPase from intracellular compartments to the plasma membrane in mammalian skeletal muscle. $\mathbf{J}$ Biol Chem 267: 5040-5043.

26. Hawkins BR, Ma JTC, Lam KSL, et al, 1985 Association of HLA antigens with thyrotoxic Graves' disease and periodic paralysis in Hong Kong Chinese. Clin Endocrinol (Oxf) 23: 245-252.

27. Yeo PPB, Chan SH, Lui KF, et al, 1978 HLA and thyrotoxic periodic paralysis. Br Med J 2: 930.

28. Tamai H, Tanaaha K, Komaki G, et al, 1987 HLA and thyrotoxic periodic paralysis in Japanese patients. J Clin Endocrinol Metab 64: 1075-1078.

29. Kung AWC, Lau KS, Fong GCY, Chan V, 2004 Association of novel single nucleotide polymorphisms in the calcium channel $\alpha 1$ subunit gene (Cav1.1) and thyrotoxic periodic paralysis. J Clin Endocrinol Metab 89: 13401345. 